\title{
Assessment of donor deferral rates and deferral reasons at the Namibia blood transfusion services
}

\author{
Martin Gonzo, Aili Shuuveni, Munyaradzi Mukesi, \\ Israel Chipare, Sylvester Moyo
}

\begin{abstract}
Aims: Despite the need to recruit and retain more blood donors, some are deferred from donating to ensure their safety and that of recipients is maintained. Examination of donor deferrals provides insights into how donor procurement strategies can be improved. Provision of safe and adequate supply of blood products is a major public health issue globally. As a result provision of safe blood should be ensured through proper donation procedures and safe transfusion. To assess the donor deferral rates and reasons for deferral among donors at Namibia blood transfusion services (NAMBTS) from o1st April 2012 to 31st March 2015. Methods: A retrospective study was conducted by reviewing the deferred donor records from 1 April 2012 to 31 March 2015. Results: Out of 50,074 prospective donors, 4302 (8.6\%) donors were deferred. The deferral rates for females and males were $74.0 \%$ and 26.0\%, respectively. Of the deferred donors
\end{abstract}

Martin Gonzo', Aili Shuuveni², Munyaradzi Mukesi ${ }^{1}$, Israel Chipare ${ }^{4}$, Sylvester Moyo ${ }^{4}$

Affiliations: ${ }^{1} \mathrm{MSc}$, Lecturer, Faculty of Health and Applied Science, Namibia University of Science and Technology, Windhoek, Namibia; ${ }^{2}$ BSc, Graduate, Faculty of Health and Applied Science, Namibia University of Science and Technology, Windhoek, Namibia; ${ }^{3} \mathrm{BSc}$, Technical Head, Namibia Blood Transfusion Service, Windhoek, Namibia; ${ }^{4} \mathrm{PhD}$, Director, Faculty of Health and Applied Science, Namibia University of Science and Technology, Windhoek, Namibia.

Corresponding Author: Martin Gonzo, Faculty of Health and Applied Sciences, Namibia University of science and Technology, P. Bag 13388, Windhoek, Namibia, E-mail: mgonzo@nust.na

Received: 25 June 2016

Accepted: 05 August 2016

Published: 24 August 2016
80.8\% were new donors and $19.2 \%$ were repeat donors. Common deferral reasons included low hemoglobin levels (45.0\%), pregnancy and breast feeding (13.7\%) and being on medication (13.6\%). Being on medication accounted for high deferral rate (24.0\%) among male donors while in female donors, low hemoglobin levels caused high (53.0\%) deferral rate. Conclusion: The deferral of donors has a negative impact on donor retention as deferred donors are less likely to return. Studying the profile of differed donors revealed that anemia is a major contributing factor and this is more pronounced in females. It may be pertinent to address the issue of anemia by educating the donor population on ways to enhance erythropoietic activity.

Keywords: Anemia, Blood transfusion, Donor deferral, Donor retention

\section{How to cite this article}

Gonzo M, Shuuveni A, Mukesi M, Chipare I, Moyo S. Assessment of donor deferral rates and deferral reasons at the Namibia blood transfusion services. Int J Blood Transfus Immunohematol 2016;5:37-43.

Article ID: 100027IJBTIMG2016

$$
* * * * * * * * *
$$

doi:10.5348/ijbti-2016-27-OA-7

\section{INTRODUCTION}

Blood transfusion is a life-saving intervention that has an essential role in patient management within health 
care systems [1]. The history of blood transfusion began with a British physician, William Harvey, who discovered the circulation of blood in 1628. The first recorded successful blood transfusion occurred in 1665 in England by Richard Lower, who transfused blood from animals to animals; followed by Jean-Baptiste Denis from Paris who transfused blood from animals to human in 1667 [2]. In 1818, a British obstetrician James Blundell performed the first successful transfusion of human blood to a patient for the treatment of postpartum hemorrhage. The discovery of the human ABO blood groups by an Austrian physician, Karl Landsteiner in 1901, identified the first major steps in understanding reactions that were caused by blood group incompatibility [3].

Most blood establishments only collect blood from voluntary non remunerated blood donors (VNRBDs) who are at low risk for transfusion transmissible Infections (TTIs), [4]. A large number of blood donors are not able to donate blood successfully as they are prohibited from donating due to several reasons. Donor deferral is initiated either by the donation centre based on information disclosed by prospective donors or by the donor through self-deferral. These deferrals occur because of the belief that a donor's behavior, exposures, or history may represent an increased risk to safety of the blood supply and as well as to the health of the donors [5].

The provision of safe and efficacious blood and blood components involves a number of processes from the selection of appropriate donor population, screening of presenting donors and testing of donated blood units [1]. Blood from the Namibia blood transfusion service (NAMBTS) was found to be safe [6].

\section{Importance of blood donation}

Blood collection and transfusion are important to the functioning of a self-sufficient community resource based health care system in the whole world at large [7]. Mostly, blood components are needed in major surgeries, traumas, accidents, burns, injuries, obstetric hemorrhages, blood replacement in neonates, prematurity, and other causes of acute and chronic anemia [8]. Cancer treatment may also create the need for blood transfusion [9]. Research done found that $10-40 \%$ of cancer patients develop anemia of chronic diseases which results from long term medical condition that could affect production and lifespan of red blood cells [10].

Developing countries receive only $39 \%$ of the world's blood supply which is donated, although, they have $82 \%$ of the global population [6]. Adequate blood supplies are the major contributory factor in preventing deaths especially among women and children [6]. In Africa, it was found that $34 \%$ of maternal deaths are caused mostly by severe hemorrhage during labor, delivery and postpartum due to inadequate blood supply [11]. In Namibia, , road crashes were registered in 2009 and statistics revealed that there has been an increase on the number of road crashes since $2002-2009$ by $5.5 \%$ thus created need for blood transfusion [12].

\section{Blood transfusion}

Blood transfusion is the practice of infusing blood intravenously to compensate for inadequate blood in the circulatory system. Blood transfusion saves lives and improves health, but millions of patients who need blood do not have access to safe blood. A better understanding of the current characteristic of the African blood transfusion system and its donors is essential for the best management of the blood supply in developing countries [13].

\section{Donor safety}

Blood donation occurs when the person gives blood with the intention of it being used on someone in need of a blood transfusion [14]. Donors' safety is managed in a way that ensures high standards of care and assures them of the concern of the blood establishment for their health and wellbeing [1]. Pre donation testing is, therefore, an important part of managing donor's safety. The testing includes ensuring donors have an hemoglobin level of at least $12.5 \mathrm{~g} / \mathrm{dL}$ before they are allowed to donate. Deferral criteria are in place to protect donors' safety especially those with low hemoglobin, pregnant/ lactating women and recent surgical procedures. Donor safety is also maintained after donation to ensure high standard of care and to assure donors that blood establishments care for their health and wellbeing [1]. Pre donation screenings also include answering the questionnaire as well as point of care testing that involves hemoglobin, blood pressure, and weight examinations.

The questionnaire is designed to ensure that it is medically safe for the donor to donate blood and to minimise the risk of transfusion transmissible infections (TTIs). It enables the donors to make informed consent and understand that their blood will be tested for TTIs and other tests [15]. In Namibia, the questionnaire includes questions on physical information, donors' health and lifestyle, and declaration.

\section{Laboratory screening}

Laboratory screening of donated blood ensures provision of safe blood, free from transfusion transmissible infections. Various types of screening assays are available and they include Immunoassay (EIAs) and nucleic acid amplification technology (NAT) assays [1]. Currently, NAMBTS uses both EIA and NAT for TTI testing. The main advantage of NAT screening is the reduction of the window period [16]. In Namibia, HIV and hepatitis B are part of the mandatory tests for all donated blood. These infections account for $7 \%$ of discarded donor blood at NAMBTS [17]. Other mandatory screening tests carried out in the laboratory are hepatitis $\mathrm{C}$ and syphilis. Hemoglobin determination 
using the copper sulfate specific gravity method as well as the HemoCue point of care testing equipment is also mandatory. Infections such as syphilis and hepatitis $\mathrm{C}$ account for $2 \%$ [17].

Common deferral reasons encountered during donor screening include low hemoglobin levels. Anemia has been reported as the main cause of deferral in studies by Bahadur et al. [18], Gajja et al. [19] and Ekwere et al. [4]. Some studies have found high blood pressure as the most common deferral reason [20, 21].

\section{MATERIALS AND METHODS}

This retrospective study was conducted by reviewing records of the donors who were deferred from donating blood from 1st April 2012 to 31st March 2015 at Namibia Blood Transfusion Services. The study included data on 50,074 blood donors that were registered during the period under study.

These blood donors were all voluntary donors nationwide that were mainly selected based on the selection criteria according to Standard for the Practice of Blood Transfusion in Namibia.

\section{Inclusion criteria and exclusion criteria}

Registered deferred donors who presented at NAMBTS during the period under study were included in the study. The study, however, excluded non-registered donors who were deferred or deferred themselves from blood donation.

\section{Sample size}

A total of 4,302 deferral donors enrolled into this study.

\section{Sampling procedure}

In this study, a purposive sampling procedure was used. Particularly, all records of donors who were deferred from donating blood were retrieved and analyzed.

\section{Data collection methods}

Donor information such as gender and deferral reasons was extracted from archives in the NAMBTS Blood Transfusion Laboratory Information System (LIS).

\section{Data analysis}

Statistical package social sciences (SPSS) version 22 and Microsoft excel were used for data analysis. Deferral rate was analyzed according to gender, donor group and causes for deferral. This data was computed into Microsoft excel and presented in tables.

\section{Ethical consideration}

The permission to conduct this study was granted by Namibian University of Science and Technology (NUST), Namibia blood transfusion service (NAMBTS) and Ministry of health and social services (MoHSS) research ethics committees.

\section{RESULTS}

The deferral rate at NAMBTS was assessed over a three-year period from 1st April 2012 to 31 March 2015. The NAMBTS uses a financial year which runs from March to April as such the results are presented were assessed using the same year format. A total number of 50,074 donors presented for blood donation at NAMBTS, out of which 4,302 (8.6\%) were deferred from donation. Table 1 gives deferral rates of whole blood donors at NAMBTS for the period April 2012 to March 2015. Of the deferred donors $26.1 \%$ were males and $74.0 \%$ were females aged from 16 to 65 years. For the donors who were deferred, $80.8 \%$ were new donors, and $19.2 \%$ were repeat donors. The most common cause for deferral was low hemoglobin (45.0\%), followed by prospective donors who were pregnant/breast feeding (13.7\%) and donors on medication (13.6\%). The year 2013 had the highest deferral rate of $10.2 \%$ while 2015 had the lowest deferral rate of $7.5 \%$ as indicated in Table 2 below. Deferral rates had a decreasing trend from 2013 to 2015.

Table 3 gives the distribution of deferral donors based on gender and it is evident that the majority of deferred donors were females. Male deferral rate decreased gradually from 2013 (428) to 2015 (338) while female deferral rates increased for the same period. New donors were found to have a high proportion of deferrals as indicated in Table 4. The least deferral rate was for the repeat donors. In an attempt, to clarify why the donors were deferred it became apparent that anemia was the main cause for donor deferral. Table 5 below indicates the deferral reasons and it shows that almost half of the deferrals were due to low hemoglobin. Of the four leading causes of deferrals by gender, Table 6 gives that anemia and taking medication were the most common reasons for deferrals in females and males respectively.

\section{DISCUSSION}

Across the world, previous studies have reported deferral rates ranging from 5.6-35.6\% [20]. The deferral rate in this study was comparable to the $10.4 \%$ found by Awasthi et al. [22] at a tertiary teaching hospital in North India. Another study done in India found the deferral rate of 5.19\% [20] and in Africa, a study done by Ekwere et al. [4] at a tertiary hospital in South Nigeria found (16\%) deferral rate and it is higher compared to the deferral rate found in this study. In Namibia, all the registered blood donors were VNRBDs. VNRBDs are considered as safe 
Table 1: Frequency of whole blood donors at NAMBTS

\begin{tabular}{lllll} 
Gender & Donors registered & No. of Deferrals & Deferral rate (\%) & Gender \\
\hline Males & 24617 & 1120 & $4.5 \%$ & Males \\
Female & 25457 & 3182 & $12.5 \%$ & Female \\
Total & 50074 & 4302 & $8.6 \%$ & Total
\end{tabular}

Table 2: Frequency of successful and deferral donors per year

\begin{tabular}{|c|c|c|c|c|}
\hline Donor group & 2013 & 2014 & 2015 & Total \\
\hline $\begin{array}{l}\text { Frequency of successful } \\
\text { Donors }\end{array}$ & $13504(89.8 \%)$ & $15545(91.7 \%)$ & $16723(92.5 \%)$ & $45772(91.4 \%)$ \\
\hline Frequency of deferred Donors & $1531(10.2 \%)$ & $1413(8.3 \%)$ & $1358(7.5 \%)$ & $4302(8.6 \%)$ \\
\hline Total & $15035(100 \%)$ & $16958(100 \%)$ & $18081(100 \%)$ & $50074(100 \%)$ \\
\hline
\end{tabular}

Table 3: Frequency of deferral donors based on gender

\begin{tabular}{lllll} 
Year & Male & Female & Total & Year \\
\hline 2013 & $428(28.0 \%)$ & $1103(72.0 \%)$ & $1531(100 \%)$ & 2013 \\
2014 & $355(25.1 \%)$ & $1058(74.9 \%)$ & $1413(100 \%)$ & 2014 \\
2015 & $338(24.9 \%)$ & $1020(75.1 \%)$ & $1358(100 \%)$ & 2015 \\
Total & $1121(26.1 \%)$ & $3181(74.0 \%)$ & $4302(100 \%)$ & Total \\
\hline
\end{tabular}

Table 4: Deferral frequency per donor category

\begin{tabular}{lcc} 
Donor category & Frequency of deferrals & (\%) \\
New donors & 3476 & 80.8 \\
Repeat donors & 826 & 19.2 \\
Total & 4302 & 100 \\
\hline
\end{tabular}

Table 5: Frequency of donor deferral reasons at NAMBTS

\begin{tabular}{lcc} 
Reasons & Frequency of deferrals & (\%) \\
Low hemoglobin & 1936 & 45.0 \\
Pregnant / Breast feeding & 590 & 13.7 \\
On medication & 584 & 13.6 \\
Medical procedures & 487 & 11.3 \\
Hypertension & 121 & 2.8 \\
\hline
\end{tabular}

Table 6: Four leading causes of deferral according to gender

\begin{tabular}{llll} 
& Male $(\mathbf{n = 1 1 2 0})$ & & Female $(\mathbf{n = 3 1 8 2})$ \\
\hline Reasons & Frequency (\%) & Reasons & Frequency (\%) \\
On medication & $269(24.0)$ & Low hemoglobin & $1688(53.04)$ \\
Low hemoglobin & $248(22.1)$ & Pregnant & $590(18.54)$ \\
Medical procedure & $237(21.1)$ & On medication & $315(9.90)$ \\
Hypertension & $86(7.70)$ & Medical procedure & $250(7.86)$ \\
\hline
\end{tabular}


donors [11] and that is why there was a low deferral rate in this study compared to the study done in Nigeria. The study done in Nigeria found that the highest numbers of donors were family replacement donors. Variations in deferral rates are caused by multiple factors such as: different donor selection criteria used by every national guideline policy in each country like weight, age, hemoglobin levels, blood donation interval, high risk sexual activities and higher incidence of first time donors.

The deferral rate has been decreasing from 2013 (10.2\%) to 2015 (7.5\%) by $2.7 \%$ this could be due to an improvement in the education system on blood donation that guide and stimulate potential blood donors to tackle the factors that could contribute to the deferral process. There is an increasing trend in the successful donation from $2013(89.8 \%)$ to 2015 (92.5\%). This showed an inverse relationship in the trends for deferral and successful donations. However, even in this study, the deferral rates were more or less the same between each specific year studied.

Among the deferral population, male represent deferral cases of 1121 (26.1\%) and female represent 3181 (74.0\%). This pattern of deferral population in males and females is similar to that reported in India, in which female donors constitute a high proportion (90.8\%) of the deferred population [18]. This also correlate with findings by [4] in Nigeria, that $34.8 \%$ were female donors while $12.4 \%$ were male donors who were deferred out of the total presented donors. Variations in deferral rate based on the gender between countries could reflect the total gender population presented for donation. Some countries have more males than females who presented for donation or vice versa. Other cases that could contribute to the high deferral rates in females could be chronic blood loss due to menstruation which has a direct effect on iron bioavailability.

Findings in this study show that new deferred donors accounted for $80.8 \%$ compared to repeat donors (19.2\%) of deferral population. This is in agreement to the results found by Birjiandi et al. [21] in Iran were first time deferred donors represented $76.5 \%$ of the total deferral populations compared to $17.5 \%$ in regular donors. A high percent in first time deferral donors and a low percentage of deferral in regular and repeat donors confirms the importance of retaining and managing donors as well as the need to educate new donors. Studies have also shown that the level of knowledge and awareness among donors and non-donors differ. Donors tend to know more about blood donation and its importance than non-donors, thus triggering the need to raise awareness among the populations [23].

From this study, low hemoglobin (45.0\%) which leads to anemia has been reported as the main cause of deferral at NAMBTS. This finding is agreeable to the outcome (40.9\%) from the research conducted by Pisudde et al., [24] in East India. In Nigeria, TTIs (50.4\%) was found to cause most of the deferrals. However, in the case of pre-donation deferral reasons hemoglobin (39.0\%) was the highest cause of donor deferral [4] which correlates with our study. Low hemoglobin is a curable condition and donors are expected to return for blood donation after a specified period of time provided that they take iron supplements so that hemoglobin level could be above $12.5 \mathrm{~g} / \mathrm{dl}$ of which is the minimum requirement at NAMBTS. Findings from this study, however, do not agree with those that were found at district transfusion centre in India as high blood pressure (39.9\%) was the leading cause of the donor deferral [20]. Similarly, results from another study done in Iran found abnormal blood pressure (36.5\%) to be the highest deferral cause [21, 25].

The second most reason causing donor deferral at NAMBTS was pregnancy/breast feeding (13.7\%). This deferral rate is high compared to $0.3 \%$ that was found by Awasthi et al. [22] in North India. Another study done in India found $0.21 \%$ rate of deferral among pregnant/ breast feeding women. Deferral rate due to lactation is low in India compared to Namibia. This could be because pregnant/breast feeding women were not given adequate information if they could donate blood.

Being on medication (13.6\%) was the third cause of deferral among donors at NAMBTS. Findings for this study are lower than for those that were found in Iran (23.3\%) by Birjiandi et al. [21].

Donors who had had medical procedures done on them (11.3\%) also contributed a high percentage of blood donor deferral reasons in Namibia. Other reasons included hypertension, tattoos, diagnostic procedures, body piercing, dental procedures, underweight, age (over 65 years), MVA, irregular pulse, hepatitis, cancer, diabetes, hypotension, tuberculosis, heart disease, accidental exposure to blood, animal bite, malaria, blood transfusion, permanent make-up and psychiatric disorder are also found to cause deferral. However, they contributed below $2.5 \%$ of the deferral population.

In this study, the highest leading cause of deferral in males was taking medication (24.0\%) while low hemoglobin $(53.0 \%)$ was found to be causing majority of deferrals in females. The finding from this study is higher than for the study done in India. In India, $6.28 \%$ of deferral due to medication was found in males and $3.75 \%$ in females. The largest cause of deferrals in males in this study does not agree to the study conducted in Delhi, India as low hemoglobin (28.7\%) was found as the largest cause of male deferrals [18]. The largest cause of deferral in females was low hemoglobin (74.1\%) in the same study [18] and that is agreeable to the results of this current study. Another study done in India found that the main cause of deferral in males (30.3\%) and females (51.0\%) was low hemoglobin [22].

There is correlation in the findings from many studies done in India as low hemoglobin was found to be causing the highest deferral in both male and female genders. 


\section{CONCLUSION}

The provision of safe and efficacious blood and blood components for transfusion involves a number of processes from the selection of appropriate donor population to the issue of compatible blood and its administration to the patient. Donors' safety is managed in a way that ensures high standards of care and assures them of the concern of the blood transfusion services for their health and well-being. The present study evaluated the donor deferral rate and reasons of donor deferral at Namibia blood transfusion services. The deferral rate was found to be $8.6 \%$ and the most common cause of deferral reason is low hemoglobin (45.0\% in females). There are differences in deferral rates from country to country and this could be due to different donor selection criteria used by each national guideline policy in each country or setting. Studying the profile of blood donors could help to identify sections of the population which could be targeted to increase the pool of voluntary blood donors and also to guide and provide the necessary essential database for the policy design and programme implementation.

$* * * * * * * * *$

\section{Acknowledgements}

We wish to acknowledge the Department of Biomedical Sciences and Namibia blood transfusion services (NAMBTS) for supporting the research.

\section{Author Contributions}

Martin Gonzo - Conception and design, Analysis and interpretation of data, Drafting the article, Critical revision of the article, Final approval of the version to be published

Aili Shuuveni - Acquisition of data, Analysis and interpretation of data, Critical revision of the article, Final approval of the version to be published

Munyaradzi Mukesi - Conception and design, Analysis and interpretation of data, Critical revision of the article, Final approval of the version to be published

Israel Chipare - Conception and design, Acquisition of data, Critical revision of the article, Final approval of the version to be published

Sylvester R. Moyo - Analysis and interpretation of data, Critical revision of the article, Final approval of the version to be published

\section{Guarantor}

The corresponding author is the guarantor of submission.

\section{Conflict of Interest}

Authors declare no conflict of interest.

\section{Copyright}

(C) 2016 Martin Gonzo et al. This article is distributed under the terms of Creative Commons Attribution License which permits unrestricted use, distribution and reproduction in any medium provided the original author(s) and original publisher are properly credited. Please see the copyright policy on the journal website for more information.

\section{REFERENCES}

1. World Health Organization. Screening Donated Blood for Transfusion Transmissible Infections. 2010. [Available at: http://www.who.int/bloodsafety/ ScreeningDonatedBloodforTransfusion.pdf]

2. Giangrande PL. The history of blood transfusion. Br J Haematol 2000 Sep;110(4):758-67.

3. Learoyd P. Historical Review: A short history of blood Transfusion; National Blood Service. Leeds Blood Centre, Scientific \& Technical Training 2006;1-18.

4. Ekwere TA, Ino-Ekanem M, Motilewa OO, Ibanga IA. Pattern of Blood Donor Deferral in a Tertiary Hospital, South-south, Nigeria: A three-year study review. International Journal Blood Transfusion Immunohematology 2014;4:7-13.

5. Gonçalez TT, Sabino EC, Schlumpf KS, et al. Analysis of donor deferral at three blood centers in Brazil. Transfusion 2013 Mar;53(3):531-8.

6. Bates I, Manyasi G, Medina Lara A. Reducing replacement donors in Sub-Saharan Africa: challenges and affordability. Transfus Med 2007 Dec;17(6):43442.

7. Shaz BH, Demmons DG, Hillyer KL, Jones RE, Hillyer CD. Racial differences in motivators and barriers to blood donation among blood donors. Arch Pathol Lab Med 2009 Sep;133(9):1444-7.

8. Davidson T, Ekermo B, Gaines H, Lesko B, Åkerlind $\mathrm{B}$. The cost-effectiveness of introducing nucleic acid testing o test for hepatitis B, hepatitis C, and human immunodeficiency virus among blood donors in Sweden. Transfusion 2011 Feb;51(2):421-9.

9. American Cancer Society. Blood Transfusion and Donation. 2013. [Available at: h t t p : / / w w w . cancer.org/treatment/ treatmentsandsideeffects/treatmenttypes/ bloodproductdonationandtransfusion/index]

10. Hassan BAR. Anemia of Chronic Diseases (ACD). Nat Prod Chem Res 2013;2:e104.

11. Bates I, Chapotera GK, McKew S, van den Broek N. Maternal mortality in Sub-Saharan Africa: The contribution of ineffective blood transfusion services. BJOG 2008 Oct;115(11):1331-9.

12. Road accidents in Namibia; National Road Statistic Report. 2013. [Available at: http://www.nrsc. org.na/files/downloads/51d_2012\%20Road\%20 Accident\%20Statistical\%20Report.pdf]

13. Tagny CT, Diarra A, Yahaya R, et al. Characteristics of blood donors and donated blood in SubSaharan Francophone Africa. Transfusion 2009 Aug;49(8):1592-9.

14. Uma S, Arun R, Arumugam P. The knowledge, attitude and practice towards blood donation among voluntary blood donors in chennai, India. J Clin Diagn Res 2013 Jun;7(6):1043-6.

15. World Health Organization. Blood Donor Counselling; Implementation Guidelines. 2014. [Available at: 
http://www.who.int/bloodsafety/voluntary_ donation/Blooddonorcounselling.pdf]

16. Mavenyengwa RT, Mukesi M, Chipare I, Shoombe E. Prevalence of human immunodeficiency virus, syphilis, hepatitis $\mathrm{B}$ and $\mathrm{C}$ in blood donations in Namibia. BMC Public Health 2014 May 5;14:424.

17. Ministry of Health and Social Services. Followup to the Declaration of Commitment on HIV/ AIDS. Namibia Country Report Directorate: Special Programme (HIV/AIDS, TB \& Malaria). 2005.

18. Bahadur S, Jain S, Goel RK, Pahuja S, Jain M. Analysis of blood donor deferral characteristics in Delhi, India. Southeast Asian J Trop Med Public Health 2009 Sep;40(5):1087-91.

19. Gajjar H, Shah FR, Shah NR, Shah CK. Whole Blood Donor Deferral Analysis at General Hospital Blood Bank - A Retrospective Study. Journal of Medical Sciences 2014;3(2):72-6.

20. Girish CJ, Chandrashekhar TN, Ramesh BK, Kantikar SM. Pre-Donation Deferral of Whole Blood Donors in District Transfusion. Journal of Clinical and Diagnostic Research 2012;6(1):47-50.
21. Birjandi F, Gharehbaghian A, Delavari A, Rezaie N, Maghsudlu M. Blood donor deferral pattern in Iran. Arch Iran Med 2013 Nov;16(11):657-60.

22. Awasthi S, Dutta S, Haritwal A, Ansari M, Arathi N, Agarwal D. Evaluation of the Reasons for Predonation Deferral of Prospective Blood donors in a Tertiary Teaching Hospital In North India. The internet journal of public health 2009;1(1):1-3.

23. Lownik E, Riley E, Konstenius T, Riley W, McCullough J. Knowledge, attitudes and practices surveys of blood donation in developing countries. Vox Sang 2012 Jul;103(1):64-74.

24. Pisudde PM, Shyam S, Rekha D, Gon S. Evaluation of Pre-donation Deferral Reason among the Blood Donors Visiting ESIC Hospital in Eastern India. J blood disorder Transfusion 2015;6(2):1-4.

25. American Red Cross, America's Blood Centers and The Armed Services Blood Program. Circular of Information for the Use of Human Blood and Blood Components. 2013. [Available at: www.aabb.org/tm/ coi/Documents/coi1113.pdf]
Access full text article on other devices

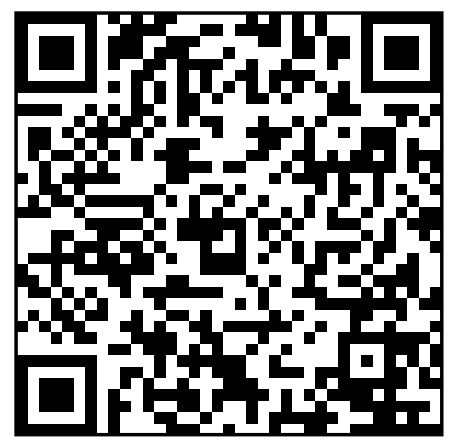

Access PDF of article on other devices

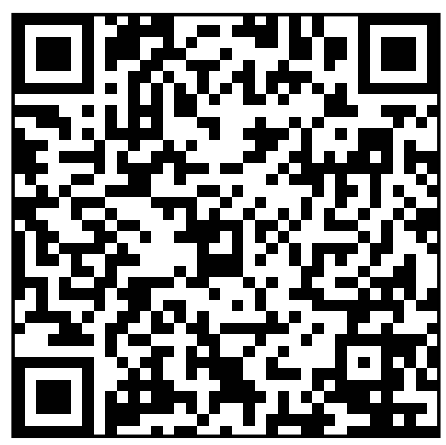

